\title{
1-S08-4 Symposium8
}

\section{CRMP2 Binding Compound Accelerates Recovery from Central Nervous System damage}

\author{
Jitsuki Susumu, Takuya Takahashi
}

Dept. Physiol., Yokohama City Univ. Grad. Sch. of Med.

Damage to the central nervous system (CNS) causes severe neurological condition, such as sustained sensory, motor, cognitive dysfunction and compromise work capacity and self-care. No pharmacological intervention that could foster recovery and complement current rehabilitation has yet been established as effective. Restoration of motor impairment after CNS damage is considered to be the result of compensative neural plasticity in spared neural circuit, and the Experience-dependent synaptic AMPA ( $\alpha$-amino-3-hydroxy-5-methyl-4-isoxazole-propionic-acid) receptor (AMPAR) delivery underlies behaviors that require neural plasticity such as learning. We found that a small compound, edonerpic-maleate (also known as T-817MA), facilitated experience-driven synaptic glutamate AMPA receptor delivery and resulted in the acceleration of motor function recovery after cortical or spinal cord cryoinjury. Edonerpic bound to collapsin-response-mediator-protein 2 (CRMP2), a downstream molecule of semaphorin, and is thought to be related to synaptic plasticity and learning. Furthermore, we detected CRMP2-dependent activation of $\mathrm{ADF} /$ cofilin by edonerpic maleate in the plasticity-inducing condition. Indicating edonerpic could facilitate synaptic AMPAR delivery through the regulation of actin dynamics. Thus, edonerpic-maleate, a neural plasticity enhancer, could be a clinically potent small compound to accelerate rehabilitation after damage of CNS. 